\section{Primeros resultados de la evaluación de un programa de Atención Temprana en síndrome de Down}

\author{
First results of the evaluation of an Early \\ Intervention program for Down's syndrome
}

\section{Resumen}

El presente estudio pretende comprobar si existen diferencias en 29 niños con síndrome de Down ( 17 niños y I 2 niñas) con edades comprendidas entre Io y I 2 meses ( $M=$ I I, $58 ; D T=$ I, 74), I 4 de los cuales no reciben tratamiento en Atención Temprana. Se utiliza el Programa para la Estimulación del Desarrollo Infantil-PEDI de Zulueta y Mollá (2006). Se intentan cuantificar los logros obtenidos (uno experimental y otro control) en el área motora, área perceptivo-cognitiva, área sociocomunicativa y área de la autonomía o de socialización tras la aplicación del programa. Los resultados muestran diferencias entre ambos grupos en tres de las cuatro áreas de evaluación, en concreto en el área perceptivocognitiva, $(t=3,26 ; p<0,05 ; d=0,95)$, lenguaje $(t=3,54 ; p<0,05$; $d=0,98)$ y autonomía/socialización $(t=4,90 ; p<0,0 \mathrm{I} ; d=0,8 \mathrm{I})$. Se discuten los resultados obtenidos y se plantean limitaciones de este estudio y perspectivas futuras para investigadores y profesionales que trabajan con niños de o a 6 años con síndrome de Down.

\section{Palabras clave:}

Atención Temprana, entrenamiento mediado, evaluación, programa de intervención, discapacidad intelectual.

\section{Abstract}

This study aims to test whether there are differences in 29 children with Down syndrome ( 17 boys and I 2 girls) aged between Io and I 2 months ( $\mathrm{M}=\mathrm{II}, 58 ; \mathrm{SD}=\mathrm{I}, 74), \mathrm{I} 4$ are control, using a training based on the mediation of learning potential and mediated learning experience. The research attempts to quantify the achievements of the two groups (one experimental and one control), in the motor, perceptual-cognitive area, the socio-communicative area and the area of autonomy and socialization. The results show differences between the groups in three of the four areas of assessment, particularly in the area perceptual-cognitive $(t=3,26 ; p<$ $0,05 ; d=0,95)$, language $(t=3,54$, $p<0,05, d=0,98)$ and autonomy/ socialization $(t=4,90 ; p<0, \mathrm{OI}$; $d=$ $\mathrm{O}, 8 \mathrm{I})$. The results, the limitations of this study and future perspectives for researchers and professionals working with children from o-6 years with Down's syndrome are discussed.

\section{Keywords}

Early Intervention, mediated training, evaluation, intervention program, intellectual disability.

\section{Ma Auxiliadora Robles-Bello <auxiaziz@yahoo.es>}

Asociación Síndrome de Down de Jaén (España)
Para citar:

Robles-Bello, M.A. (20I6): "Primeros resultados de la evaluación de un programa de Atención Temprana en síndrome de Down”, Revista Española de Discapacidad, 4 (I): 53-65.

Doi: <http://dx.doi.org/IO. $5569 / 234$ O5 I04.04.0I.03>

Fecha de recepción: I7-OI-20I6 Fecha de aceptación: 3I-05-20I6 
Introducción

La atención temprana (AT) en España ha evolucionado rápidamente en los últimos treinta años gracias a las administraciones públicas, a las asociaciones de padres y madres de niños con discapacidad y a los investigadores y profesionales dedicados a este ámbito (Candel, 2005; Grupo de Atención Temprana-GAT, 2000, 2008a; Quirós, 2009). La Federación Estatal de Asociaciones de Profesionales de Atención Temprana-GAT (2004) propone tres niveles de actuación en Atención Infantil Temprana: la promoción y prevención primaria dirigida a la población infantil en general; la prevención secundaria, formando parte de los programas sanitarios, educativos y sociales dirigidos a los grupos de riesgo, y la prevención terciaria, interviniendo con niños con trastornos del desarrollo y sus familias. De ahí, que el tipo de población incluida en los programas de AT sea muy heterogéneo (GAT, 2008a; GAT, 20I I; Robles-Bello y Sánchez-Teruel, 20I Ia, 20I3; Sánchez-Teruel, 20I I).

En España esta atención se materializa en los Centros de Desarrollo Infantil y Atención Temprana (CDIAT) que según la GAT (2008b, 2OII) son servicios de carácter interdisciplinar destinados a la población comprendida entre cero y seis años que presentan alguna alteración o disfunción en su desarrollo, o riesgo de padecerla. Trabajan en la creación de programas de Atención Temprana en coordinación con centros concertados o subvencionados con la Administración y de manera intrainstitucional entre educación, sanidad y servicios sociales. Su principal función es la de potenciar al máximo las capacidades del niño o la niña para así lograr una adaptación familiar, escolar y social. El equipo profesional que atiende al niño o niña y a la familia, y se coordina con la Administración, es o debe ser interdisciplinar y transdisciplinar (GAT, 2000).

Si nos centramos en la Atención Temprana de una población concreta como es el síndrome de Down (SD) encontramos que según la
Organización Diagnóstica para la Atención Temprana-ODAT, basada en una de las clasificaciones internacionales diagnósticas (Organización Mundial de la Salud-OMS, I992), este síndrome se clasifica en el Eje I: Factores Biológicos de Riesgo, I.a. Prenatal, I.a.h. cromosomopatías y síndromes dismórficos. Debe ser atendido desde estos programas porque son objeto de tratamiento desde la prevención terciaria los niños con alteraciones o discapacidad documentada, así en este grupo se incluyen a los niños con retrasos, alteraciones o discapacidades de tipo cognitivo, de la movilidad, de la comunicación o sensoriales. Con estos niños se debe iniciar la intervención educativa desde el mismo momento del nacimiento o desde el momento en que se detecta el déficit.

Este síndrome sigue siendo la principal causa de discapacidad intelectual y la alteración genética humana más común: I/800 nacimientos vivos para ambos sexos (Robles-Bello y SánchezTeruel, 20 I Ib). Sin embargo el número de programas de Atención Temprana diseñados para ellos y con cierto rigor científico es bastante limitado (Candel, 2000). Uno de ellos es el de Candel (2000), aplicado en población heterogénea con discapacidad intelectual (Candel et al., I98 5 ; Hanson, 2004; CluniesRoss, I979; Cunningham, I988; Dmitriev, I988; Hanson y Schwarz, I978; Sharav y Shlomo, I986; Ludlow y Allen, I979; Dykens y Kasari, I998; Fidler, 2005), pero que se ha implementado de manera tradicional en el SD a través del documento (Candel, 2002).

Hoy en día no se pone en duda la eficacia general de la AT (Candel, I998, 2000; Galeote et al., 201 2; Flórez, I991; Gibson y Harris, I998; Hanson, 2004; Spiker y Hopmann, I997).

Sin embargo, parece que los datos en niños con discapacidad proceden, generalmente, de estudios con una baja calidad metodológica (Candel, 2000, 2002). De los 57 trabajos revisados por Dunst (I988) sobre programas de AT, sólo el $5 \%$ usaban un diseño verdaderamente experimental. Esto nos lleva necesariamente a ser más prudentes a la hora de exagerar los beneficios que produce la 
AT, ya que las limitaciones metodológicas reducen la fiabilidad de los datos aportados (Candel, 2000). En líneas generales, elaborar conclusiones tan rotundas, cuando existe una gran heterogeneidad de las muestras examinadas y cuando los autores informan de resultados positivos cuando se intentan enseñar destrezas curriculares específicas, reduce fiabilidad y validez desde el punto de vista científico. Los avances son más limitados en áreas de desarrollo más generales, sobre todo en las esferas cognitivas. Algunos trabajos han confirmado los efectos de la AT como elemento impulsor del progreso motor de los niños con parálisis cerebral y como elemento estimulador del apoyo y aceptación por los padres. Sin embargo, los datos que indican la eficacia de la AT en los niños con minusvalías motoras son escasos, pues se han centrado en examinar los efectos del tratamiento únicamente sobre las distintas variables motoras.

Pese a ello aún faltan investigaciones que valoren la eficacia de determinados programas de AT que se están poniendo en marcha en España en poblaciones específicas con síndrome de Down. Las personas que trabajamos en estos CDIAT entendemos que existen muchos aspectos a mejorar en AT, uno de ellos es la gran variedad de las ofertas en la intervención desde estos programas (European Agency for Development in Special Needs Education, 2005) que no distingue entre discapacidades o problemáticas diferentes en función de los niños y, aunque en la actualidad hay una corriente importante de estos trabajos en niños prematuros (Piñero et al., 20I4) no encontramos trabajos que prueben la eficacia de determinados programas de intervención que se están llevando a cabo con niños con SD en España.

De ahí, que el objetivo de este estudio fuese conocer si los niños y niñas con síndrome de Down sometidos a un programa concreto de atención temprana como es el Programa para la Estimulación del Desarrollo Infantil-PEDI de Zulueta y Mollá (PEDI) (2006), presentan diferencias respecto a aquellos otros en los que su intervención en este programa no es sistemática.

\section{Método}

\section{- Participantes}

La muestra estuvo formada por 29 niños con SD que al comenzar la evaluación contaban con una edad de entre Io y I 2 meses ( $M=$ I I, 58 ; $D T=1,74)$. Tras la intervención durante seis meses, sus edades se situaban entre los I 6 y I 8 meses.

Toda la muestra fue dividida en dos grupos: un grupo denominado experimental (GE de aquí en adelante) formado por I 5 niños con síndrome de Down, 8 niños y 7 niñas. El domicilio habitual de estos niños y niñas se encontraba en una ciudad que cuenta con varios centros gratuitos de Atención Infantil Temprana, al que asistían de forma regular $(4 \mathrm{O}$ 5 veces por semana). El otro grupo, denominado grupo control (de aquí en adelante GC), estaba compuesto por I4 niños con síndrome de Down. Este grupo estaba constituido por 9 niños y 5 niñas cuyo tratamiento en AT con el PEDI era poco sistemático y tardío debido a la gran distancia entre su domicilio habitual, zona de sierra con carreteras mal comunicadas, y el centro de AT más próximo, que distaba entre 90-100 Km. A esta distancia es necesario añadir el inconveniente de transitar por dichas zonas en período invernal. Todos los niños y niñas participantes en este estudio presentaban en su cariotipo la Trisomía 2 I, una de las tres formas de expresión del síndrome de Down, y fueron diagnosticados al nacer por el servicio de pediatría de salud pública más cercano a su lugar de residencia.

\section{- Instrumentos}

- Brunet-Lézine Revisado (Escala de Desarrollo Psicomotor de la Primera Infancia, Brunet-Lézine, I997). Su objetivo es la evaluación del nivel madurativo del niño en las cuatro áreas que explora: control postural/motor, coordinación óculo-motriz (cognición-percepción), 
lenguaje/comunicación, sociabilidad/ autonomía. Permite obtener una edad de desarrollo y un cociente de desarrollo global del niño, así como una valoración parcial de la edad de desarrollo y del cociente de desarrollo del niño en cada una de las áreas exploradas. La validez interna de la escala respecto al cociente de desarrollo global sobre niños de 6 meses es de 0,64 y en niños de I 2 meses de 0,58 . La fiabilidad test-retest de todas las subdimensiones en niños de 6 meses se encuentra entre 0,50 y 0,92 y para la escala global es de 0,89 . La fiabilidad test-retest en niños de $\mathrm{I} 2$ meses se encuentra entre , 79 y 0,8 I y para la puntuación global es de $\circ, 8$ I. Esta prueba es aplicable entre los $\circ$ y los 30 meses de edad.

- Programa para la Estimulación del Desarrollo Infantil-PEDI de Zulueta y Mollá (2006): Este programa para la estimulación del desarrollo es aplicable desde los o hasta los 4 años y presenta, ordenadas evolutivamente en un inventario, las principales adquisiciones del desarrollo del niño a lo largo de sus primeros años. El programa diseñado por estos autores incluye: inventario del desarrollo, fichas de estimulación en las áreas motora, perceptivo-cognoscitiva, de lenguaje y social, y material didáctico. La información obtenida en la anamnesis, junto con la derivada de la aplicación de las pruebas y escalas de desarrollo constituye la base para la programación y puesta en marcha de los objetivos de intervención. Esta propuesta consiste en atender dentro del programa las necesidades de aprendizaje en las que el niño presenta los mayores déficits. Por tanto, en el PEDI se contempla la necesidad de establecer un programa individual con cada sujeto, ajustado a su edad, nivel y necesidades del niño dirigido a potenciar las áreas cognitiva, motora, autonomía y de comunicación. Asimismo, enfatiza la participación activa de la familia durante todo el proceso de intervención. Las sesiones son impartidas individualmente con una duración de 45 minutos y llevadas con recursos variados y metodología adecuada a cada niño. El Anexo I presenta un extracto de algunas actividades recomendadas en el PEDI.

\section{- Diseño}

El diseño seguido en este estudio ha sido cuasiexperimental con un grupo de niños elegido según su diagnóstico (presentar en su cariotipo trisomía 2I-síndrome de Down), y edad (entre los ro y 12 meses de edad al inicio del estudio). Los dos grupos definidos en esta investigación (GE y GC) se establecieron por la asistencia o no del niño a un centro de AT de forma regular y continuada. Los datos obtenidos se analizaron con el paquete estadístico SPSS I9,0. Los análisis consistieron en un contraste de medias relacionadas a través a la prueba $\mathrm{T}$ dirigida a conocer las posibles diferencias existentes en la fase pre-test y post-test de las cuatro áreas evaluadas, así como la medición del tamaño del efecto en caso de existir dichas diferencias. Debido a las implicaciones éticas de la investigación, no es posible asignar de manera aleatoria a los niños a un grupo o a otro sino que se hace en función de un hecho muy concreto que es su zona de origen, ello no debe invalidar el estudio ya que tradicionalmente es conocido que las características del síndrome en cuestión no dependen de su zona de origen sino del síndrome en sí mismo (Pueschel, r988a, I988b, I995, 2002).

\section{- Procedimiento}

Los niños y niñas del GE se incluyeron como participantes cuando fueron derivados al CDIAT. Se contactó con los padres de los niños en la consulta de acogida (primera consulta) de una asociación de SD y en ese momento los padres decidían su participación o no en el estudio. En la primera consulta de acogida se confirmaba con los padres que los niños y niñas no hubiesen participado antes en algún otro tipo de programa de atención infantil temprana. En función del lugar de residencia de los niños y de la regularidad de asistencia al CDIAT fueron 
asignados a cada uno de los grupos (GE o GC). Una vez obtenido el consentimiento de los padres se pasó a realizar la evaluación.

El esquema general de actuación con cada niño del GE participante en el estudio es la siguiente:

En primer lugar se evaluó (evaluación pre-test) cada niño y niña con la Escala de Desarrollo Brunet-Lézine (I997). Posteriormente se aplicó un tratamiento, el PEDI de Zulueta y Mollá (2006) y se valoraron los objetivos en las diferentes áreas (motora, perceptivo-cognitiva, lenguaje y social/ autonomía) según las necesidades identificadas en los niños en la evaluación pre y las condiciones establecidas en el PEDI. Esta intervención se mantuvo durante 6 meses. Tras este periodo, se volvió a evaluar (evaluación post-test) a través de la Escala de Desarrollo de Brunet-Lézine (I997) contabilizando los objetivos conseguidos tras la aplicación del programa PEDI de Zulueta y Mollá (2006). La secuencia seguida con los niños participantes en el GC es la misma a la descrita con la excepción de que no existe intervención a través del PEDI. Todas las sesiones de evaluación (GE y GC) y las sesiones de intervención (solo GE) se realizaron de forma individualizada por psicólogos expertos en el PEDI que llevan trabajando en su aplicación más de cinco años.

\section{Resultados}

En primer lugar, se comprobó que no existían diferencias entre los dos grupos de sujetos que componen la muestra en relación con la edad y sexo. El hecho de que no existiese relación entre los grupos en edad $\left(F_{(\mathrm{I}, 28)}=0,435 ; p=0,6 \mathrm{I} 6\right) \mathrm{y}$ sexo $\left(\mathrm{RV}_{(2)}=4,038 ; p=0, \mathrm{I} 33\right)$ muestra que la distribución es homogénea en estas variables en los dos grupos.

El primer objetivo consistió en comprobar si hay diferencias en la ejecución de los niños que componen el grupo experimental entre las condiciones de pre-test y post-test en las áreas intervenidas. Los resultados (tabla I) muestran que existen diferencias significativas entre los resultados de las evaluaciones pre-test y post-test en tres de las áreas intervenidas, concretamente: perceptivo-cognitiva, $(t=3,26 ; p<0,05)$, lenguaje $(t=3,54 ; p<0,05)$ y autonomía/ socialización $(t=4,90 ; p<0, \mathrm{OI})$. En todos los casos, el nivel de ejecución en el nivel post-test es superior al nivel de ejecución mostrado en el nivel pre-test. Los resultados en relación con la cuarta área intervenida, el área motora, a pesar de que existen diferencias éstas no alcanzan el nivel de significación estadística.

\begin{tabular}{l}
\hline Tabla 1. Resultados evaluación pre-post intervención en el grupo experimental \\
\begin{tabular}{|l|c|c|c|c|}
\hline \multicolumn{4}{|c|}{ Grupo Experimental } \\
\hline Áreas & $\begin{array}{c}\text { PRE } \\
\text { M(DT) }\end{array}$ & $\begin{array}{c}\text { POST } \\
\text { M(DT) }\end{array}$ & $t$ & Sig. \\
\hline Motora & $4.80(2.03)$ & $5.20(3.02)$ & 4.02 & $.65 \mathrm{~ns}$ \\
\hline Perceptivo-cognitiva & $1.45(1.15)$ & $8.69(3.84)$ & 3.26 & $.03^{\star}$ \\
\hline Lenguaje & $.41(.62)$ & $6(5.45)$ & 3.54 & $.02^{*}$ \\
\hline Social/Auto & $.30(.50)$ & $3.94(1.97)$ & 4.90 & $.00^{\star *}$ \\
\hline
\end{tabular}
\end{tabular}

*Diferencias significativas $(\mathrm{p}<0,05)$; *Diferencias muy significativas $(\mathrm{p}<0, \mathrm{OI})$;

Diferencias no significativas ( $p=n s$ )

Fuente: elaboración propia. 
Los resultados para el grupo Control (tabla 2) muestran que no existen diferencias significativas en el nivel de ejecución mostrada por los niños entre la evaluación previa y la evaluación realizada a los 6 meses, en ninguna de las áreas examinadas.

Respecto a la existencia de diferencias entre los resultados pre y post en ambos grupos (tabla 3), parece que se detecta un importante desarrollo en el grupo experimental respecto al grupo control fundamentalmente en las áreas perceptivo-cognitiva, lenguaje y social/autonomía debido a la aplicación del tratamiento en el grupo experimental respecto al grupo control. Existe ganancia en todas las áreas, siendo más evidente en el área de la socialización y en la del lenguaje.

Tabla 2. Resultados evaluación momento 1 y 2 en el grupo control (no hay intervención)

\begin{tabular}{|l|c|c|c|c|}
\hline & \multicolumn{3}{|c|}{ Grupo Control } \\
\hline Áreas & $\begin{array}{c}\text { Evaluación } \\
\text { momento } \\
\mathbf{1} \\
\mathbf{M}(\mathbf{D T})\end{array}$ & $\begin{array}{c}\text { Evaluación } \\
\text { momento } \\
\mathbf{2} \\
\mathbf{M} \text { (DT) }\end{array}$ & $\boldsymbol{t}$ & Sig. \\
\hline Motora & $4.4(3.2)$ & $4.22(2.24)$ & 1.02 & $.65 \mathrm{~ns}$ \\
\hline Perceptivo-cognitiva & $1.93(2.77)$ & $2.20(2.14)$ & 1.26 & $.30 \mathrm{~ns}$ \\
\hline Lenguaje & $.21(.31)$ & $.47(.12)$ & .37 & $.19 \mathrm{~ns}$ \\
\hline Social/Auto & $.49(.68)$ & $.60(1.18)$ & .90 & $.20 \mathrm{~ns}$ \\
\hline
\end{tabular}

*Diferencias significativas $(\mathrm{p}<.05)$; **Diferencias muy significativas $(\mathrm{p}<.0 \mathrm{I})$; Diferencias no significativas $(p=\mathrm{ns})$

Fuente: elaboración propia.

Tabla 3. Resultados grupo experimental y control y diferencias entre ambos grupos pre-post

\begin{tabular}{|c|c|c|c|c|c|c|c|c|c|}
\hline \multirow[b]{2}{*}{ Áreas } & \multicolumn{2}{|c|}{ Grupo Experimental } & \multicolumn{2}{|c|}{ Grupo Control } & \multicolumn{2}{|c|}{$\begin{array}{c}\text { Diferencia } \\
\text { pre-1 } \\
\text { (ambos } \\
\text { grupos) }\end{array}$} & \multicolumn{2}{|c|}{$\begin{array}{c}\text { Diferencia } \\
\text { post-2 } \\
\text { (ambos } \\
\text { grupos) }\end{array}$} & \multirow{2}{*}{$\begin{array}{c}\begin{array}{c}\text { Potencia } \\
\text { del } \\
\text { efecto } \\
\text { post-test }\end{array} \\
\begin{array}{c}d \text { de } \\
\text { Cohen }\end{array}\end{array}$} \\
\hline & $\begin{array}{l}\text { PRE } \\
\text { M(DT) }\end{array}$ & $\begin{array}{l}\text { POST } \\
\text { M(DT) }\end{array}$ & $\begin{array}{l}\text { Evaluación } \\
\text { momento } 1 \\
\text { M(DT) }\end{array}$ & $\begin{array}{c}\text { Evaluación } \\
\text { momento } 2 \\
\text { M(DT) }\end{array}$ & $t$ & Sig. & $t$ & Sig. & \\
\hline Motora & $3.80(2.03)$ & $5.20(3.02)$ & $4.4(3.2)$ & $4.22(2.24)$ & 2.35 & $.23 n s$ & 1.23 & $.06 n s$ & .39 \\
\hline $\begin{array}{l}\text { Perceptivo- } \\
\text { cognitiva }\end{array}$ & $1.45(1.15)$ & $8.69(3.84)$ & $1.93(2.77)$ & $2.20(2.14)$ & 1.45 & $.90 \mathrm{~ns}$ & 1.97 & $.00^{\star \star}$ & .95 \\
\hline Lenguaje & $.41(.62)$ & $6(5.45)$ & $.21(.31)$ & $.47(.12)$ & .89 & $.50 \mathrm{~ns}$ & 3.12 & $.00^{\star *}$ & .98 \\
\hline Social/Auto & $.30(.50)$ & $3.94(1.97)$ & $.49(.68)$ & $.60(1.18)$ & .23 & $.65 n s$ & .42 & $.02^{*}$ & .81 \\
\hline
\end{tabular}

*Diferencias significativas $(\mathrm{p}<.05)$; **Diferencias muy significativas $(\mathrm{p}<.0 \mathrm{I})$; Diferencias no significativas $(p=\mathrm{ns})$

Fuente: elaboración propia. 


\section{Discusión}

Este estudio tenía como objetivo principal valorar la eficacia de un programa de AT, el PEDI, en niños que presentan Síndrome de Down y cuyas edades se sitúan en fechas próximas al comienzo de la intervención entre los ro y los I 2 meses de edad. De forma más específica, este estudio examina las ganancia en cuatro áreas intervenidas, (motora, perceptivo-cognitiva, lenguaje y social/ autonomía). Los resultados muestran que existen ganancias en todas esas áreas tras la intervención a través del PEDI y es mantenida durante 6 meses. Esas ganancias alcanzan el nivel de significación estadística en tres de las cuatro áreas intervenidas, específicamente las áreas perceptivo-cognitiva, lenguaje y social/autonomía. En estas áreas, la mejora en la ejecución asociada a la intervención es especialmente relevante en el área social, donde los niños adquieren tras la intervención el mayor número de objetivos planteados inicialmente.

La ausencia de una mejora significativa en la ejecución en el área motora puede estar asociada a una de las características fenotípicas que tradicionalmente aparecen en los niños con SD, la hipotonía (Bull, 20 I I; National Down Syndrome Society, 20I2). Sin embargo, en los estudios tradicionales de Gibson y Harris (I998) los mayores beneficios se encontraban en la motricidad fina, autoayuda y los peores resultados se daban en las áreas de lenguaje, cognitiva y motricidad gruesa. En cualquier caso, este estado hipotónico nos sugiere que puede ser necesario reforzar la intervención en el área motora en el plan de intervención desarrollado a través del PEDI.

Tal y como lo han planteado algunos autores (Iacono et al., 2010) respecto a las diferencias obtenidas en el área social, están muy relacionadas con la ganancia que se ha obtenido también en el área del lenguaje, ya que al mejorar la comunicación en un niño con SD también es probable que mejore la socialización. Especialmente interesante es el hecho de que existen diferencias entre el grupo experimental y control que parecen ser debidas al tratamiento implementado y que seguramente no son consecuencia del paso del tiempo o de la edad ya que esas diferencias se mantienen en el segundo momento de medida entre ambos grupos a favor del grupo experimental, con excepción del área motora, la cual parece ser la única área que avanza independientemente del tratamiento con la PEDI.

Igualmente, resulta interesante que los efectos de la intervención se mantengan seis meses después ya que siguen existiendo diferencias entre el GE y el GC en el segundo momento de la intervención, lo cual es contrario a los datos tradicionales de Gibson y Harris (I998) que indicaban que la intervención temprana era eficaz a corto plazo, con una tendencia a que los beneficios logrados decaigan con el tiempo hasta los niveles de los niños con SD del grupo control, o los niveles de los niños con SD que evolucionaron espontáneamente.

La escasa muestra utilizada nos lleva a plantear los resultados de este estudio como un estudio piloto sobre la efectividad de un programa de intervención con niños con SD más que como un programa que es efectivo en todos los niños con esta discapacidad. Investigaciones futuras podrían implementar este programa en una muestra más amplia de niños y niñas ya que la AT se dirige a un amplio rango de edades (o-6 años) y este estudio se ha dirigido hacia niños de muy corta edad, entre los IO-I 2 meses de edad. De ahí que una de las limitaciones de este estudio sea comprobar la eficacia del programa PEDI en niños tan pequeños. Sería interesante realizar este estudio en niños cuyo rango de edad sea más amplio, pero resulta muy interesante comprobar la eficacia de este tipo de tratamientos en un rango de edad especialmente susceptible a la estimulación en los niños pequeños.

En el futuro, se hace necesaria la implementación de programas de intervención en diversas edades en niños y niñas con la misma discapacidad, para obtener resultados con mayor valor clínico dentro del rango de edad de la AT en España (o-6 años). 
Asimismo, y dado el formato de evaluación en los momentos pre y post de este estudio cuasi-experimental, se podría considerar que la intervención materna o paterna puede haber alterado los resultados en relación con el nivel de ejecución de los niños. Pese a eso, los datos obtenidos en el Grupo Control y donde se aplica el mismo protocolo de evaluación no sugieren cambios significativos entre ambos momentos de la evaluación, por lo que podríamos considerar que esa posible intervención paterna en los resultados haya sido controlada.

A pesar de que el síndrome de Down es la población con discapacidad más estudiada a nivel internacional (Bull, 20I I; National Down Syndrome Society, 20I 2) y que participa en los programas de Atención Temprana desde finales de los años 90 debido a su alta incidencia, a que es detectado muy tempranamente y a que es etiológicamente homogéneo (Wishart, 2002), la realización de este trabajo ha podido hacer reflexionar sobre qué programa o programas de intervención son los más adecuados para este tipo de discapacidad intelectual en España (GAT, 20II). Además, se ha observado que no existen muchas investigaciones españolas que comparen la efectividad de programas tradicionales en contrapartida con otros programas de entrenamiento más innovadores en SD (Candel, I993, I998; Robles-Bello y Sánchez-Teruel, 2013). Futuras investigaciones podrían ir en esta línea para ofrecer a las administraciones competentes y a los profesionales de la Atención Temprana programas de intervención basados en la evidencia y contextualizados según la discapacidad que presente el niño o la niña. 


\section{Anexo 1}

Ejemplo de implementación del PEDI (Zulueta y Mollá, 2006) en las cuatro áreas de intervención. Fuente: Zulueta y Mollá (2006).

\section{- Área motora:}

- Supina: pasar de la posición de costado a boca arriba; mantenerse en posición simétrica; pasar a la posición de costado; mantener los pies en alto para jugar con ellos; cogerse los pies; apoyar los pies y empujarse hacia atrás; voltear para conseguir algo; llevarse los pies a la boca; moverse en un círculo de $360^{\circ}$.

- Prona: pasar el brazo por delante; levantar la cabeza $45^{\circ}$ y girarla a uno y otro lado; mantener la cabeza levantada $90^{\circ}$, apoyado en los antebrazos; mover la cabeza arriba, abajo y a los lados; apoyarse en las manos, teniendo los brazos extendidos; pasar a boca arriba; girar en círculo; mantenerse de rodillas, sentado sobre sus talones con mínima ayuda; reptar; situarse en posición de gateo, balancearse y elevar un brazo; gatear; pasar a sentado; pasar de la posición de gateo a ponerse de pie con apoyo; gatear sobrepasando obstáculos.

- Sedestación: mantener la cabeza al elevarle de los brazos; controlar la cabeza en cualquier posición; mantenerse sentado con apoyo y recuperar la posición al desequilibrarse; ayudar activamente al sentarle; mantenerse sentado con apoyo de las dos manos; extender los brazos para protegerse de la caída; mantenerse sentados sin ayuda un minuto; apoyar las manos lateralmente para evitar las caídas; pasar de sentado a de pie, con poca ayuda; pasar a boca abajo; mantenerse sentado sin perder el equilibrio; sentarse con ayuda de un soporte; pasar a la posición de gateo; pasar de boca arriba a estar sentado sin ayuda.
- Posición de pie: sostener parte de su peso; hacer movimientos de flexión-extensión de piernas; mantenerse de pie, cogiéndose a las manos de un adulto un minuto; dar algunos pasos con ayuda; mantenerse de pie, apoyado en un mueble; levantar un pie y bajarlo de nuevo, estando apoyado a un apoyo externo; andar apoyado en los muebles; sentarse, desde la posición de pie; andar llevándole de dos manos; agacharse a coger algo, estando de pie con apoyo; mantenerse de pie sin ningún apoyo un minuto; andar de una mano; dar tres o cuatro pasos solo.

\section{- Área perceptivo-cognitiva:}

- Visión: fijar la mirada en personas y objetos; seguir trayectorias horizontales de personas y objetos; seguir los desplazamientos de una persona situada a un metro; mirar alternativamente dos objetos; mirarse las manos; seguir trayectorias verticales y circulares; mirar hacia atrás; volver los ojos para mirar un objeto que aparece lateralmente; mirar el objeto que sostiene; fijar la mirada en un objeto pequeño; seguir los desplazamientos de una persona por una habitación; sostener un objeto mientras mira otro; seguir todo tipo de trayectorias con objetos pequeños; observar sus pies al moverlos; seguir movimientos rápidos de personas y objetos.

- Audición: reaccionar a un sonido moviendo las piernas y/o los brazos; localizar sonidos laterales; localizar sonidos por debajo de su cabeza; localizar sonidos por encima de su cabeza; hacer sonar los objetos que sostiene; localizar sonidos en cualquier dirección; producir sonidos con instrumentos.

- Miembros superiores: llevarse las manos al rostro y a la boca; abrir las manos frecuentemente; coger al contacto; llevarse 
objetos a la boca; cogerse las manos y juguetear con ellas; tocar objetos; coger objetos; coger objetos de la mesa con movimientos de barrido; individualizar sus brazos; golpear objetos verticalmente; pasarse los objetos de mano; coger objetos de la mesa con movimientos desde el codo; coger con oposición del pulgar; golpear dos objetos horizontalmente; coger un tercer objeto soltando uno de los que tenga; lanzar objetos voluntariamente; coger objetos con los dedos índice y pulgar; explorar con el dedo índice.

- Sacar-meter: sacar objetos de recipientes; meter objetos en recipientes; sacar aros de soportes; meter aros en soportes.

- Permanencia del objeto: encontrar objetos caídos; quitarse pantallas de su cara; encontrar un juguete oculto por una pantalla; anticipar los movimientos de objetos y personas.

- Uso de medios: utilizar un soporte fijo para obtener un objeto; utilizar un cordón para obtener un objeto.

- Imitación: imitar gestos en juegos y canciones sencillas; realizar gestos, sin verlos previamente, en juegos y canciones sencillas.

\section{- Área del lenguaje:}

- Lenguaje comprensivo: responder a su propio nombre; responder al "no"; comprender palabras asociadas a situaciones cotidianas; localizar por su nombre a personas y objetos familiares; utilizar de forma simbólica y funcional objetos familiares.

- Lenguaje expresivo: emitir sonidos indiferenciados; balbucear sonidos silábicos en cadena y cortados; Seguir los turnos en la emisión de sonidos; emitir sonidos referidos a objetos o acciones; expresar deseos con gestos; decir una palabra con significado.

- Área social:

- Alimentación: succionar regularmente; utilizar los labios para vaciar la cuchara; tomar semisólidos con la cuchara; sujetar el biberón con las manos; movimientos verticales de masticación; beber de un vaso sin atragantarse; masticar alimentos; comer con los dedos; control de babeo

- Conductas interactivas: reconocer y reaccionar a estímulos emocionales; sonrisa social; reírse a carcajadas; reconocer las caras familiares; vocalizar cuando se le cuida; anticipar situaciones; tender los brazos al adulto al pedírselo; juguetear ante el espejo; extender los brazos para que lo cojan; demostrar miedo ante una persona extraña; jugar él solo un momento. 
Bull, M.J. (20I I): "Committee on Genetics. Health supervision for children with Down syndrome". Pediatrics, I28: 393-406.

Candel, I. (2005): "Elaboración de un programa de atención temprana". Revista Electrónica de Investigación Psicoeducativa, 7: I 5 I-I92.

Candel, I. (2002): "Programa de Atención Temprana. Intervención en niños con Síndrome de Down y otros problemas de desarrollo", en Candel, I. (ed.): Atención Temprana. Situación actual y perspectivas de futuro. Madrid: CEPE.

Candel, I. (2000): Atención Temprana: Eficacia de los programas de atención temprana en niños con sindrome de Down. Apuntes sobre la eficacia de los programas de AT. Investigación en niños con sindrome de Down, Madrid: Federación Española de Síndrome de Down.

Candel, I. (1998): Atención temprana. Niños con sindrome de Down y otros problemas de desarrollo, Madrid: Federación Española del Síndrome de Down.

Candel, I. (1993): Programa de atención temprana. Intervención en niños con sindrome de Down y otros problemas de desarrollo, Madrid: CEPE.

Candel, I. et al. (1985): Una experiencia de estimulación temprana en niños con sindrome de Down (en línea). <http://www. papelesdelpsicologo.es/vernumero.asp?id=2 I 9>, acceso 27 de septiembre de 2013 .

Clunies-Ross, G.G. (I979): “Accelerating the development of Down's Syndrome infants and Young children". Journal of Special Education, I3: I69-I77.

Cunningham, C.C. (I988): "Intervención temprana: algunos resultados del estudio del grupo síndrome de Down en Manchester", en Flórez, J. y Troncoso, M.V. (eds.): Síndrome de Down: Avances en Acción Familiar. Santander: Fundación Síndrome de Down de Cantabria.

Dmitriev, V. (I988): “Cognition and the acceleration and maintenance of developmental gains among children with Down syndrome: Longitudinal data". Down's Syndrome, I I: 6-II.

Dunst, C.J. (I998). "Sensorimotor development and developemental disabilities", en Burack, J.A. et al. (eds.): Handbook of Mental Retardation and Development. New York: Cambridge University Press.

Dunst, C.J. (I990): "Sensorimotor development of infants with Down syndrome", en Cicchetti, D. y Beeghley, M. (eds.): Down Syndrome. The Developmental Perspective. New York: Cambridge University Press.

Dykens, E.M. y Kasari, C. (I998): “Problemas de conducta en muchachos con síndrome de Down, síndrome de Prader-Willi y retraso intelectual inespecífico". Revista Síndrome de Down, I 5: 17-23.

European Agency for Development in Special Needs Education (2005): Atención Temprana. Análisis de las situaciones en Europa. Aspectos clave y recomendaciones. Informe Resumen (en línea). <https://www.european-agency.org/ sites/default/files/early-childhood-interventionanalysis-of-situations-in-europe-key-aspectsand-recommendations_eci_es.pdf>, acceso $8 \mathrm{de}$ junio de 2016 .

Fidler, D.J. (2005): “The Emerging Down Syndrome Behavioural Phenotype in Early Childhood Implications for Practice”, en Candel, I. (2010): Atención Temprana en niños con Sindrome de Down. Madrid: Federación Española de Síndrome de Down.

Flórez, J. (I99I): “La realidad biológica del síndrome de Down”, en Flórez, J. y Troncoso, M.V. (dir.): Sindrome de Down: Avances en Acción Familiar. Santander: Fundación Síndrome de Down de Cantabria.

Galeote, M. et al. (2012): Atención conjunta $y$ desarrollo del vocabulario en niños con sindrome de Down, en AELFA (ed.): Comunicación presentada en el XVIII Congreso 
Internacional de la Asociación Española de Logopedia, Foniatría y Audiología. Madrid: Asociación Española de Logopedia, Foniatría y Audiología.

GAT (2OI I): La realidad actual de la Atención Temprana en España, Madrid: Real Patronato sobre Discapacidad.

GAT (2008a): La realidad de la Atención Temprana en España, Madrid: Real Patronato sobre Discapacidad.

GAT (2008b): Organización Diagnóstica para la Atención Temprana. Manual de Instrucciones, Madrid Real Patronato sobre Discapacidad.

GAT (2004): Organización Diagnóstica para la Atención Temprana. Manual de Instrucciones, Madrid: Real Patronato sobre Discapacidad.

GAT (2000): Libro Blanco de la Atención Temprana, Madrid: Real Patronato de Prevención y Atención a Personas con Minusvalía.

Gibson, D. y Harris, A. (I988): “Aggregated early intervention effects for Down's syndrome persons: patterning and longevity of benefits". Journal of Mental Deficiency Research, 32: I-I7.

Hanson, M.J. (2004): "Veinticinco años de Intervención Temprana. Un seguimiento de niños con síndrome de Down y sus familias”. Revista Sindrome de Down 2I: 42-53.

Hanson, M.J. y Schwarz, R.H. (I978): "Results of a longitudinal intervention program for Down's Syndrome infants and their families". Education and Training of Mentally Retarded, 4: 403-407.

Iacono, T. et al. (2010): "Relationships amongst age, language and related skills in adults with Down syndrome". Research in Developmental Disabilities, 3 I: 568-576.

Ludlow, J.R. y Allen, L.M. (I979): “The effect of early intervention and pre-school estimulus on the development of the Down's syndrome child". Journal of Mental Deficiency Research, 23: 29-44.

National Down Syndrome Society (2012): Down syndrome clinics and health care providers database (en línea). <http://www.ndss. org/Resources/Health-Care/Health-CareProviders/>, acceso 27 de septiembre de 2013.

Organización Mundial de la Salud, OMS (I992): Clasificación Estadística Internacional de Enfermedades y Problemas relacionados con la Salud-CIE-Io, Madrid: Mediator.

Piñero, J. et al. (20I4): Atención Temprana en el Ámbito Hospitalario, Madrid: Pirámide.

Pueschel, S.M. (2002): Síndrome de Down: Hacia un futuro mejor. Guía para padres, Barcelona: Editorial Masson.

Pueschel, S.M. (I995): “Características físicas de las personas con síndrome de Down”, en Perera, J. (dir.). Sindrome de Down. Aspectos específicos. Barcelona: Masson.

Pueschel, S.M. (1988a): Psysical characteristics, chromosome análisis, and treatment approaches in Down syndrome, Boston: College-hill Press/ Little, Brown\&Co.

Pueschel S.M. (1988b): "Visual and auditory processing in children with Sown Syndrome", en Nadel, L. (ed.): The Psychobiology of Down Syndrome. Cambridge: The MIT press.

Quirós, V. (2009): "Nuevo modelo para la atención temprana en Andalucía: directrices y desafíos”. Revista Iberoamericana de Educación, 48: I-8.

Robles-Bello, M.A. y Sánchez-Teruel, D. (20I3): “Atención Infantil Temprana en España”. Papeles del Psicólogo, 34 (2): I32-I 43.

Robles-Bello, M.A. y Sánchez-Teruel, D. (coord.) (20I Ia): Evaluación e Intervención en Atención Infantil Temprana: Hallazgos recientes y casos prácticos, Jaén: Publicaciones de la Universidad de Jaén.

Robles-Bello, M.A. y Sánchez-Teruel, D. (20 I Ib): ¿Qué es el sindrome de Down? Actualización en Sindrome de Down: Creer para ver, Jaén: Fortalezas Editorial.

Sánchez-Teruel, D. (20I I): "Las enfermedades raras en niños de o-6 años: una aproximación clínica”, en Robles-Bello, M.A. y SánchezTeruel, D. (coord.): Evaluación e Intervención en Atención Infantil Temprana: Hallazgos 
recientes y casos prácticos. Jaén: Servicio de Publicaciones de la Universidad de Jaén.

Sharav, T. y Shlomo, L. (I986): "Stimulation of infants with DownSíndrome: Long-term effects". Mental Retardation, 24: 8I-86.

Spiker, D. y Hopmann, M.R. (I997): “The effectiveness of early intervention for children with Down syndrome", en Guralnick, M.J. (ed.): The Effectiveness of Early Intervention. Baltimore: Paul H. Brooks.

Wishart, J. (2002): "Learning in young children with Down syndrome: Public perceptions, empirical evidence", en Cuskelly, M. et al. (eds.): Down syndrome across the life span. London: Whurr Publishers.

Zulueta, M.I. y Mollá, M.T. (2006): Programa para la estimulación del desarrollo infantil, Madrid: Cepe. 